\title{
Adaptação à pós-graduação stricto sensu: uma revisão sistemática de literatura
}

\author{
Anelise Schaurich dos Santos - Universidade Federal de Santa Maria, Santa Maria, Brasil \\ Clándia Maria Perrone - Universidade Federal de Santa Maria, Santa Maria, Brasil \\ Ana Cristina Garcia Dias - Universidade Federal de Santa Maria, Santa Maria, Brasil
}

\begin{abstract}
Resumo
Esta pesquisa teve como objetivo investigar exploratoriamente os fatores pessoais, relacionais e institucionais que são descritos na literatura como influências no processo de adaptação de estudantes de pós-graduação stricto sensu aos seus cursos. Realizou-se uma busca sistemática de estudos nacionais e internacionais sobre adaptação à pós-graduação publicados nos últimos 10 anos, por meio da combinação de diversos descritores. Optou-se por pesquisar artigos revisados por pares em periódicos científicos indexados no Portal de Periódicos da CAPES. Foram encontradas 177 publicações internacionais. Após a submissão dos artigos aos critérios de inclusão e exclusão, restaram 15 estudos, os quais constituíram a amostra final desta pesquisa. Constatou-se que a integração com a vida de pós-graduando depende, principalmente, do estabelecimento de uma relação gratificante entre orientador e orientando, ambientação com as normas e demandas do programa de pós-graduação e segurança financeira. Contudo, a temática carece de uma investigação mais aprofundada, principalmente em relação ao cenário da pós-graduação brasileira. Palavras-chave: ajustamento acadêmico; estudantes de pós-graduação; pós-graduação.
\end{abstract}

\section{Adjustment to graduation: a systematic review of literature}

\begin{abstract}
The aim of this research is to investigate in an exploratory way personal, relational and institutional factors that are described in literature as influences in the graduate students' adjustment to their courses. A systematic search of national and international studies about adjustment to graduate degree from the last 10 years through a combination of several descriptors was realized. We have chosen to search peer-reviewed articles in scientific indexed journals at Portal de Periódicos of CAPES. 177 international publications were found. After the submission of the articles to the inclusion criterions, remaining 15 studies, which constituted the final sample of this research. It was concluded that graduate life's integration depends mainly on establishing a rewarding relationship between advisor and advisee, setting the rules and demands of graduate and financial security. However, the issue needs further investigation, especially in the scenario for Brazilian graduate students.

Keywords: students adjustment; graduate students; graduate.
\end{abstract}

Adaptación al postgrado: una revisión sistemática de literatura

\begin{abstract}
Resumen
Esta investigación tuvo como objetivo analizar los factores personales, relacionales e institucionales que son descriptos en la literatura como influyentes en el proceso de adaptación de estudiantes de postgrado stricto sensu a sus carreras. Por medio de la combinación de diversos descriptores se realizó una búsqueda sistemática de estudios nacionales e internacionales, publicados en los últimos 10 años. Se investigaron artículos en periódicos científicos indizados al Portal de Periódicos de CAPES. Fueron encontradas 177 publicaciones internacionales. Una vez los artículos sometidos a los criterios de inclusión y exclusión, restaron 15 estudios, constituyendo la muestra final de esta investigación. Se constató que la integración de los estudiantes a la vida de postgrado depende principalmente de la construcción de una buena relación entre tutor/alumno, ambientación con las normas y demandas del programa de postgrado, así como también seguridad económica. Sin embargo, hace falta una investigación más profunda sobre el tema, principalmente refiriéndose al postgrado brasileño.

Palabras-clave: ajuste académico; alumnos de postgrado; postgrado.
\end{abstract}

A pós-graduação stricto sensu compõe o topo da pirâmide do sistema de ensino em âmbito mundial (Steiner, 2005). Esse nível de formação é um empreendimento relativamente recente no Brasil e tardio na história da construção do sistema educacional do país (Menandro, Tourinho, Bastos, \& Yamamoto, 2013; Tourinho \& Bastos, 2010). Sua criação data de 1965, ano em que as principais características da pós-graduação do país foram fixadas pelo parecer 977, denominado Parecer Newton Sucupira, aprovado pelo
Conselho Federal de Educação em 03 de dezembro de 1965 (Balbachevsky, 2005). Nesse parecer, a pós-graduação stricto sensu é definida como o ciclo de cursos sistematicamente organizados que dão segmento aos estudos do Ensino Superior (Conselho Federal de Educação [CFE], 1965), no qual são diferenciados dois estágios: o mestrado e o doutorado (Balbachevsky, 2005). Neste estudo, o termo "pós-graduação" será utilizado para se referir unicamente à pós-graduação stricto sensu. 
Apesar de a pós-graduação brasileira ser recente, todas as áreas do conhecimento se desenvolveram nos últimos anos. No período de 1976 a 2004, o crescimento de programas de pós-graduação recomendados pela Coordenação de Aperfeiçoamento de Pessoal de Nível Superior (CAPES) foi de aproximadamente 5,6\% ao ano (Lo Bianco, Almeida, Koller, \& Paiva, 2010). De acordo com as informações mais atuais da CAPES, no ano de 2013 estavam matriculados 219.987 estudantes nos cursos de pós-graduação no Brasil e foram tituladas 65.428 pessoas, dado que inclui os cursos de mestrado acadêmico, mestrado profissional e doutorado. Além disso, nesse mesmo ano, o Brasil totalizou 3.486 cursos de pós-graduação em seu território (Coordenação de Aperfeiçoamento de Pessoal de Nível Superior [CAPES], 2013).

A decisão por ingressar em um curso de mestrado e/ou doutorado é complexa e individual (Holley \& Caldwell, 2012). Os fatores que influenciam a entrada dos alunos na pós-graduação, que os mantêm em seus cursos e o que contribuem para a conclusão deles ainda são pouco investigados e conhecidos (Holley \& Caldwell, 2012; Tobbell \& O'Donnell, 2013). Isso acontece porque são raras as pesquisas que estudam a transição da graduação para a pós-graduação apesar da riqueza de estudos sobre as transições educacionais (Hoffman \& Julie, 2012; Tobbell \& O’Donnell, 2013). Todavia, sabe-se que, assim como em outras transições acadêmicas (por exemplo, a transição do ensino médio para o ensino superior), o ingresso na pós-graduação configura-se como uma experiência singular na vida dos estudantes (Hoffman \& Julie, 2012).

Alguns discentes não conseguem responder às exigências impostas pela pós-graduação (Hyun, Quinn, Madon, \& Lustig, 2006), a saber, cobranças por produtividade, exigência de leitura e escrita, preocupação com prazos, conciliação dos compromissos acadêmicos com a vida pessoal (Bujdoso \& Cohn, 2008; Faro, 2013; Malagris et al., 2009; Santos \& Alves Júnior, 2007; Schwartz-Mette, 2009) e relação insatisfatória com o orientador (Krauss \& Ismail, 2010; Noy \& Ray, 2012; Velho, 2005). Outros não conseguem se manter no sistema devido à falta de condições financeiras (Holley \& Caldwell, 2012; Tobbell \& O'Donnell, 2013), pois, mesmo quando recebem bolsa de estudos, o valor não é suficiente para sustentá-los (Maia, 2014). Quando uma dessas situações ocorre, a maioria dos discentes que se encontra nessa circunstância evade de seu curso (Holley \& Caldwell, 2012; Terrell, Snyder, \& Dringus, 2009; Tobbell \& O’Donnell, 2013).
No Brasil, as taxas de evasão dos cursos de pós-graduação são diferentes para cada Instituição de Ensino Superior (IES), mas podem chegar a 11,5\%, realidade presente na Universidade de São Paulo (USP) entre os anos de 2009 e 2013 (Maia, 2014). Já nos Estados Unidos, as taxas de evasão dos cursos de pós-graduação tendem a variar de $40 \%$ a 50\% (Terrell et al., 2009). A evasão na pós-graduação é decorrente do abandono do curso, do desligamento devido a reprovações consecutivas em disciplinas e, principalmente, do não término da dissertação ou tese (Velho, 2005). A discrepância entre a taxa de ingresso e a taxa de conclusão dos cursos de pós-graduação é um indicativo de que os discentes enfrentam desafios de transição ao se envolverem com o mestrado ou o doutorado (Hoffman \& Julie, 2012).

Os índices de desistência desses cursos tendem a estar relacionados ao insucesso na adaptação a esse nível de formação. Acredita-se que os mestrandos e doutorandos com maior probabilidade de abandonarem seus cursos são aqueles que não conseguiram se adequar ao contexto da pós-graduação e, assim, atender às necessidades conferidas por esse novo contexto de formação, visto que a adaptação acadêmica corresponde ao quão bem os estudantes conseguem atender às demandas acadêmicas (Feldt, Graham, \& Dew, 2011; Teixeira, Dias, Wottrich, \& Oliveira, 2008). A adaptação acadêmica é influenciada por três grandes fatores: condições pessoais (fatores pessoais), grupos de interação (fatores relacionais) e características institucionais (fatores institucionais) (Credé \& Niehorster, 2012; Feldt et al., 2011; Igue, Bariani, \& Milanesi, 2008; Polydoro, Primi, Serpa, Zaroni, \& Pombal, 2001). Os fatores pessoais englobam o bem-estar físico e o equilíbrio psicológico dos estudantes. Os fatores relacionais são compreendidos pelas relações interpessoais que os discentes estabelecem com pares, professores e familiares. Já os fatores institucionais estão associados ao contexto acadêmico experienciado pelos estudantes, isto é, ao ambiente da instituição e do curso (Almeida \& Ferreira, 1999; Baker $\&$ Siryk, 1984). Assim, o objetivo deste estudo foi investigar exploratoriamente os fatores pessoais, relacionais e institucionais que são descritos na literatura como influências no processo de adaptação de estudantes de pós-graduação stricto sensu aos seus cursos.

\section{Método}

Este estudo caracteriza-se como uma revisão sistemática de literatura. Optou-se pela utilização dessa 
abordagem metodológica porque ela permite a avaliação e a sistematização de evidências de distintas questões e de diversos tipos de pesquisa, possibilitando o acesso de maneira organizada e fidedigna ao maior número de estudos sobre determinada temática. A busca sistematizada não admite a supervalorização de trabalhos que vão ao encontro das hipóteses iniciais dos pesquisadores e a omissão de estudos que apresentem outras visões sobre o fenômeno a ser estudado (Costa \& Zoltowski, 2014).

Em setembro de 2014, realizou-se uma busca de estudos nacionais e internacionais sobre fatores que influenciam a adaptação à pós-graduação stricto sensu publicados nos últimos 10 anos. Optou-se por pesquisar artigos revisados por pares em periódicos científicos indexados no Portal de Periódicos da CAPES (portal que integra diversas bases de dados), no qual é possível acessar o texto completo das publicações disponíveis (Costa \& Zoltowski, 2014). Na opção "data de publicação", selecionou-se a alternativa "últimos 10 anos". Na opção "tipo de material”, selecionou-se a alternativa "artigos". Considerou-se a opção "no assunto" para a realização da busca, visto que não interessava a recuperação de artigos com os descritores escolhidos no título ou no autor. Também se selecionou a opção "é (exato)" para os descritos compostos (por exemplo, estudantes de pós-graduação/graduate students), uma vez que as demais opções buscavam artigos que continham uma ou outra palavra que integrava o descritor. Os descritores foram definidos a partir da consulta das palavras-chave na terminologia em Psicologia (www.bvs-psi.org.br), já que essa ferramenta é constantemente atualizada por meio da inserção de novos descritores (Costa \& Zoltowski, 2014).

A busca final utilizou a seguinte combinação de descritores: "estudantes de pós-graduação/graduate students" e "ajustamento acadêmico/students adjustment, ou saúde mental/mental health, ou amizade/friendship, ou interação interpessoal/interpersonal interaction, ou interação social/social interaction, ou pais/parents ou supervisor/supervisor, ou instituições de ensino super/ higher education institutions, ou atividades extracurriculares/extracurricular activities". Optou-se por utilizar descritores que nomeassem os aspectos que são entendidos na literatura como variáveis que influenciam a adaptação a novos contextos educacionais (Credé \& Niehorster, 2012; Feldt et al., 2011; Igue, Bariani, \& Milanesi, 2008; Polydoro et al., 2001).

A busca foi embasada em critérios de inclusão e exclusão previamente determinados pelas autoras.
Decidiu-se que os critérios de inclusão para participar da amostra final deste estudo seriam: (1) ser um estudo empírico, (2) a amostra ser constituída apenas por estudantes de pós-graduação stricto sensu, (3) relacionar alguma das dimensões (pessoal, relacional ou institucional) da adaptação acadêmica aos estudantes de pós-graduação stricto sensu, (4) apresentar a influência do fator estudado pelo artigo na adaptação dos discentes aos seus cursos. O critério de exclusão utilizado foi se tratar de um estudo intercultural, ou seja, abordar o ajustamento à pós-graduação de estudantes que não estavam estudando em seu país de origem.

Ao total, foram encontrados 177 artigos internacionais revisados por pares por meio da combinação dos descritores. O cruzamento dos termos graduate students e students adjustment recuperou 56 artigos, dos termos graduate students e mental health recuperou 56 artigos, dos termos graduate students e frienship recuperou 12 artigos, dos termos graduate students e supervisor recuperou 48 artigos, e dos termos graduate students e extracurricular activities recuperou cinco artigos. As demais combinações, bem como o cruzamento dos termos em português, não recuperou nenhum artigo.

Após a leitura dos resumos, 159 artigos foram descartados por não respeitar um ou mais critérios de inclusão ou se enquadrarem no critério de exclusão. Além disso, três artigos estavam repetidos. Assim, a amostra final foi constituída por 15 publicações internacionais, as quais foram lidas na íntegra.

\section{Resultados e Discussão}

Foram analisados 15 estudos internacionais recuperados do Portal de Periódicos da CAPES. Optou-se por agrupar os resultados dos artigos analisados em três categorias, as quais foram nomeadas: (1) fatores pessoais da adaptação à pós-graduação, (2) fatores relacionais da adaptação à pós-graduação e (3) fatores institucionais da adaptação à pós-graduação. Essa divisão foi baseada na literatura especializada sobre adaptação acadêmica, a qual entende a integração de aspectos pessoais, relacionais e institucionais como a principal influência em processos de adaptação a novos contextos educacionais (Credé \& Niehorster, 2012; Feldt et al., 2011; Igue et al., 2008; Polydoro et al., 2001).

Acredita-se que as pesquisas internacionais revisadas auxiliam na investigação da realidade vivenciada por estudantes de pós-graduação de maneira geral, uma vez que algumas dificuldades podem ser enfrentadas no mestrado e/ou no doutorado independentemente 
do país de origem do estudante. Contudo, diferenças sociais, culturais e educacionais de cada país não devem ser ignoradas no entendimento da adaptação de discentes aos cursos de pós-graduação. Por essa razão, buscou-se apresentar semelhanças e diferenças entre os resultados expostos nas categorias, característicos de sociedades culturalmente distintas do Brasil, e dados obtidos por meio de estudos nacionais. As categorias citadas serão descritas e discutidas a seguir.

\section{Fatores pessoais da adaptação à pós-graduação}

Os fatores pessoais apresentados nessa categoria englobam estudos que tratam do estado físico e psicológico dos estudantes de pós-graduação (Almeida \& Ferreira, 1999; Baker \& Siryk, 1984), os quais tendem a ser caracterizados como uma população vulnerável devido aos efeitos dos problemas físicos e psicológicos causados por elevados níveis de estresse (Schwartz-Mette, 2009). Apesar desse entendimento, a maioria dos estudos sobre a saúde de acadêmicos é executada com estudantes de graduação, o que faz com que os dados sobre o bem-estar de pós-graduandos sejam fragmentados e esparsos (Kernan, Bogart, \& Wheat, 2011). Todavia, sabe-se que a quantidade de universitários que se encontram em sofrimento psíquico é cada vez maior, além de haver um aumento da gravidade dos problemas mentais apresentados por essa população (Hyun et al., 2006). A realização de pesquisas que mapeiem a ocorrência e identifiquem as causas do mal-estar dentre mestrandos e doutorandos é importante, uma vez que problemas mentais não tratados contribuem para o abandono da pós-graduação (Hyun et al., 2006).

Os resultados do estudo de Hyun et al. (2006) com 3.121 estudantes de mestrado e doutorado dos Estados Unidos indicaram que 44,7\% dos respondentes haviam se sentido estressados em algum momento dos 12 meses anteriores à pesquisa e 57,7\% relataram que algum colega reconheceu ter se sentido estressado no mesmo período. Além disso, 46,3\% dos pós-graduandos disseram que se percebiam sobrecarregados e $39,6 \%$ afirmaram que se sentiam esgotados "frequentemente" ou "o tempo todo". As causas relatadas pelos estudantes para as percepções de estresse, sobrecarga e esgotamento foram pressão para pesquisar e dar aula, necessidade de publicar e preocupação com as condições financeiras, além de ter de lidar com a frequente ambiguidade de expectativas do orientador.

Outra pesquisa realizada com 263 doutorandos de duas universidades australianas revelou que o estresse enfrentado por pós-graduandos seguidamente advém da necessidade de gerir três aspectos: relações afetivas, demandas intelectuais e responsabilidades financeiras. A administração adequada dessas dimensões depende da habilidade para lidar com as situações complexas que se apresentam na vida, da capacidade de persistir e se revigorar em tempos de desafio e da motivação para se envolver em atividades que demandam esforço mental sobre um período de tempo prolongado. Todas essas habilidades são individuais e cada sujeito possui uma maneira única para desenvolvê-las (Cantwell, Scevak, Bourke, \& Holbrook, 2012).

A pesquisa desenvolvida por Kernan et al. (2011) também relaciona o estresse de doutorandos ao ambiente da pós-graduação, pois descreve que a maioria dos estudantes, além de enfrentar desafios financeiros, confronta-se com altos níveis de exigência acadêmica. Longas horas de trabalho, falta de apoio por parte do programa de pós-graduação, intensa concorrência entre os pares e excessiva cobrança por parte dos professores são fatores do contexto acadêmico com os quais pós-graduandos, com frequência, deparam-se e devem aprender a conviver. Esse estudo identificou que essas características, muitas vezes, desencadeiam doenças respiratórias, dificuldades interpessoais, sofrimento psicológico, problemas para dormir, uso de substâncias psicoativas e alergias. Evidências estatisticamente significativas confirmaram que tais problemas de saúde impactaram negativamente na vida acadêmica dos participantes da pesquisa, uma vez que discentes que apresentavam adversidades na saúde e preocupações psicossociais relataram dificuldades de aprendizagem e déficit de atenção (Kernan et al., 2011).

Por outro lado, o trabalho de Prepiczka e Balkin (2010) revelou que os doutorandos em Orientação Educacional de uma universidade norte-americana apresentaram escores mais altos que o restante da população no aspecto "bem-estar". As dimensões que apresentaram escores mais elevados, nesse estudo, foram "autocuidado" e "interação social". Entretanto, o "bem-estar físico" apresentou o escore mais baixo, o que pode indicar que os estudantes sacrificam a prática de exercícios físicos e a alimentação adequada para cumprir com as obrigações da pós-graduação. Mesmo assim, a maioria dos participantes dessa pesquisa acreditava que o doutorado é uma experiência mais benéfica do que estressante.

Outro aspecto de influência na adaptação a pós-graduação é a provável conquista da independência por parte dos pós-graduandos. A independência é parte integrante da habituação à pós-graduação, uma 
vez que mestrandos e doutorandos podem se sentir livres para direcionar suas escolhas e para encontrar motivação para concluir a dissertação ou a tese. Além disso, muitos pós-graduandos conquistam independência em relação aos pais, pois passam a receber o próprio "salário", isto é, a bolsa de estudos. Contudo, muitos estudantes são forçados a serem mais independentes do que imaginam devido à falta de pessoas que os direcionem (Gardner, 2010).

Não foram localizados estudos com mestrandos e doutorandos brasileiros que enfocassem aspectos pessoais positivos da pós-graduação, como as pesquisas de Prepiczka e Balkin (2010) e de Gardner (2010). Portanto, não é possível afirmar quais são as vivências benéficas possibilitadas pela pós-graduação para pós-graduandos do Brasil. Entretanto, acredita-se que a conquista da independência financeira não é comum entre mestrandos e doutorandos do país, dependendo da região onde o estudante reside. Isso acontece porque nem todos os discentes recebem bolsas de estudos e, aqueles que as recebem, tendem a não conseguir arcar com todas as suas necessidades apenas com o valor delas (Maia, 2014).

Já os resultados das pesquisas sobre estresse em pós-graduandos brasileiros (Faro, 2013; Malagris et al., 2009; Santos \& Alves Júnior, 2007) vão ao encontro dos estudos realizados por Cantwell et al. (2012), Hyun et al. (2006) e Kernan et al. (2011). O estresse em pós-graduandos brasileiros está diretamente relacionado às cobranças pelos altos níveis de produtividade acadêmica. A necessidade de dedicação exclusiva, sem um apoio financeiro significativo, a ênfase na publicação internacional e a concorrência desleal devido à falta de estabilidade profissional podem levar a um estado de esgotamento mental que é prejudicial à saúde de mestrandos e doutorandos (Malagris et al., 2009; Santos \& Alves Júnior, 2007). Somado a isso, a preocupação em não conseguir cumprir prazos e a possibilidade de não atingir o desempenho esperado pela banca, o aproveitamento nas disciplinas, o calendário e o horário de aulas são elementos que também mobilizam negativamente os alunos (Malagris et al., 2009; Santos \& Alves Júnior, 2007). Assim, o estresse em pós-graduandos advém da necessidade de conciliar exigências acadêmicas, trabalho, lar e vida pessoal, além do dever de lidar com o prazo de conclusão dos cursos (Bujdoso \& Cohn, 2008).

\section{Fatores relacionais da adaptação à pós-graduação}

Sabe-se que, para se adaptar ao contexto universitário, o discente deve socializar-se, isto é, aprender a adotar os valores, habilidades, atitudes, normas e conhecimentos necessários para a adesão a uma determinada sociedade, grupo ou organização (Gardner, 2010). Experiências gratificantes nos contextos educacionais tendem a depender da constituição de laços satisfatórios com os atores envolvidos nesse ambiente, como familiares, pares e professores (Almeida \& Ferreira, 1999; Baker \& Siryk, 1984). O êxito na socialização é indispensável para o sucesso na pós-graduação, visto que a escolha por evadir do mestrado ou doutorado comumente é atribuída à socialização pobre ou inapropriada (Gardner, 2010), conforme demonstram os resultados a seguir.

As entrevistas realizadas por Gardner (2010) com 60 estudantes de doutorado de seis programas de pós-graduação de diferentes áreas de uma universidade dos Estados Unidos revelaram que os pares são uma fonte de apoio importante para os pós-graduandos. Os colegas, por possivelmente encontrar-se em situações de vida semelhantes, conseguem entender uns aos outros e se ajudar na resolução de problemas. A autora notou que, nas áreas em que a porcentagem de doutorandos que concluem a pós-graduação nessa universidade é baixa, como a matemática e as engenharias, os entrevistados não se referiram aos pares como fonte de apoio. Isso indica que o suporte emocional e, em alguns casos, técnico oferecido pelos demais estudantes do curso auxilia na finalização da tese. Além disso, constatou-se que os processos de socialização são diferentes em cada área do conhecimento. Por exemplo, a experiência de socialização nas ciências exatas, que tende a ser baseada no trabalho de laboratório, seguidamente realizado em grupo, é diferente da experiência de socialização de alunos que recebem bolsas de estudos independentes, como frequentemente acontece na área das humanas. Há um indicativo de que o convívio diário auxilia no desenvolvimento de relações de companheirismo (Gardner, 2010), pois a criação do sentimento de pertença ao grupo é facilitada, conforme foi identificado na pesquisa brasileira de Teixeira et al. (2008).

Também a respeito do convívio diário com os pares, os pós-graduandos que participaram do estudo de Tobbell e O'donnell (2013) relataram sentir necessidade de despender algum tempo com seus colegas a fim de compartilharem experiências e trocarem conselhos. Essa vontade parece estar relacionada ao fato de que o ajustamento a novas circunstâncias é facilitado pelas relações de afetividade saudáveis que o indíviduo estabelece. Os efeitos dos eventos estressores que não podem ser evitados tendem a ser mais brandos e o prejuízo à 
saúde psicológica é menor quando o estudante percebe que tem a quem recorrer. Assim, as relações de afetividade e de atenção fazem com que o indivíduo sinta-se seguro, o que contribui para a sensação de coerência e controle sobre sua vida, fatores que causam impactos positivos na adaptação a situações desconhecidas (Souza, 2010).

Entretanto, alguns pós-graduandos da pesquisa conduzida por McAlpine (2012) com 80 estudantes de doutorado em quatro universidades do Reino Unido e do Canadá revelaram que não identificaram relevância para sua identidade e para o seu desenvolvimento profissional ao estabelecerem relações de amizade com seus pares. Alguns entrevistados ainda descreveram que não se sentiam confortáveis para construir relações de afeto no ambiente acadêmico, uma vez que acreditavam que essa é uma atividade estratégica, a qual visa benefícios profissionais, a saber, o estabelecimento de uma rede de contatos. Esses discentes revelaram que buscavam apoio emocional na família e nos amigos adquiridos em períodos anteriores ao ingresso na pós-graduação para suportarem as dificuldades encontradas no doutorado.

De fato, o apoio para suportar o desgaste do doutorado, muitas vezes, advém das relações de confiança constituídas antes da entrada no curso. De acordo com 33 doutorandos do Reino Unido, amigos de infância, familiares, colegas de graduação e colegas de trabalho são figuras importantes no desenvolvimento da motivação e servem como inspiração na tarefa de escrever uma tese. Portanto, mesmo que novos relacionamentos sejam desenvolvidos durante a pós-graduação, o que é relevante para a adaptação do estudante nesse nível de formação, os discentes frequentemente recorrem a pessoas significativas em suas vidas que não fazem parte desse contexto (Hopwood, 2010).

Além do estabelecimento de afinidade com os pares ser importante para vivências gratificantes na pós-graduação (Gardner, 2010; Tobbell \& O'donnell, 2013), a relação satisfatória de pós-graduandos com os membros do corpo docente, principalmente com o orientador, é entendida pela maioria dos mestrandos e doutorandos como um dos aspectos mais importantes da qualidade de sua experiência nesse nível de formação (Noy \& Ray, 2012). Os pós-graduandos que não se adaptam à forma e ao ritmo de trabalho de seus orientadores tendem a evadir de seus cursos. A relação de trabalho gratificante entre pós-graduandos e seus orientadores é reconhecida como otimizadora dos processos de supervisão, finalização do grau de pós-graduação, produção de teses e dissertação com maior nível de qualidade, performance adequada dos estudantes em pesquisas e satisfação na pós-graduação (East, Bitchener, \& Basturkmen, 2012; Ives \& Rowley, 2005; Holley \& Caldwell, 2012; Unswortha, Turnerb, Williams, \& Piccin-Houle, 2010).

O estudo de Bennett, Mohr, BrintzenhofeSzoc e Saks (2008), com 72 estudantes de mestrado de uma universidade de Washington, baseou-se na premissa de que o orientando transmite para a sua relação com o orientador as formas de apego que estabelece em outros relacionamentos íntimos. Por exemplo, alunos de pós-graduação, muitas vezes, procuram proximidade ou intimidade com seus supervisores em busca de conforto e esclarecimento sobre o seu trabalho quando se sentem angustiados ou perdidos. Ademais, quando os alunos percebem que são apoiados por seus orientadores, eles são mais propensos a desenvolver comportamentos exploratórios para aperfeiçoar novas habilidades. Contudo, a hipótese de que o padrão de apego do discente determina o estilo de supervisão e a intimidade da relação orientador-orientando não se confirmou. Na verdade, a qualidade dessa relação depende de características de ambos os envolvidos. De maneira geral, os estudantes que relataram um forte vínculo com o orientador atribuíram as seguintes características a ele: amigável, solícito, perspicaz, comprometido e focado.

Uma vez que o estabelecimento de afinidade entre orientando e orientador decorre de características tanto de um quanto de outro, é relevante que ambos se envolvam na escolha dessa orientação. A pesquisa realizada por Ives e Rowley (2005) fornece indícios de que essa é uma ideia verídica. Dentre os 21 pós-graduandos australianos que participaram do estudo, os discentes que se sentiram envolvidos na seleção de seus supervisores relataram satisfação com a supervisão e progrediram na escrita da dissertação ou tese. Os estudantes que escolheram seus supervisores o fizeram baseados na combinação de aspectos acadêmicos e pessoais. Isso pode causar dúvidas, uma vez que o professor e o aluno podem desenvolver uma boa relação, mas não apresentar afinidade teórica. De qualquer forma, indica-se que a seleção do orientador seja realizada por meio de critérios de afinidade de pesquisas e do grau de intimidade que o orientando espera da relação. É relevante que orientadores e orientandos apresentem padrões acadêmicos, de trabalho e métodos de pesquisa semelhantes (Ives \& Rowley, 2005).

Entretanto, não há um modelo padrão de garantia da qualidade das supervisões, que pode ser aplicado 
em todas as situações e em todas as áreas do conhecimento. Uma supervisão eficaz se esforça para atender às necessidades dos diferentes alunos, programas $\mathrm{e}$ estruturas administrativas (Egan, Stockley, Brouwer, Tripp, \& Stechyson, 2009). Também é importante que o orientador apresente conhecimento sobre o campo de pesquisa, interesse em atender às demandas acadêmicas do orientando e disponibilidade para apoiar o aluno, além de balancear elogios e críticas dirigidos aos pós-graduandos (Ives \& Rowley, 2005). O nível de satisfação apresentado pelos orientandos com a supervisão de orientadores que tentavam seguir esses pressupostos era alto no estudo conduzido por Egan et al. (2009) com 1.335 estudantes de pós-graduação de uma universidade do Canadá. Além disso, o tempo de dedicação dos orientadores para a supervisão agradava a maioria dos participantes.

A supervisão também é beneficiada quando o orientador fornece um feedback a respeito do trabalho do orientando para este, uma vez que a supervisão é um importante componente do ambiente de ensino-aprendizagem da universidade. A supervisão poderia ser definida como conversas de aprendizagem (East et al., 2012). Cinquenta e três pós-graduandos de três programas diferentes de seis universidades da Nova Zelândia relataram que recebiam feedback a respeito do conteúdo (indicação de literatura, relevância da literatura), da organização (revisão da estrutura, cortar informações desnecessárias) e da linguagem (escrita científica, pontuação, gramática) de seus trabalhos. Além disso, as formas de feedback eram: escrita seguida de reunião, somente escrita ou somente falada (East et al., 2012). Todavia, o que o aluno espera com frequência é diferente do que é proporcionado pelo orientador, o que pode causar tensão na relação e perda da eficácia do trabalho. Por isso, é importante conhecer, por meio de evidências empíricas, como a relação supervisor-aluno é construída no ambiente da pós-graduação e a eficácia do feedback para essa constituição (East et al., 2012).

De forma complementar, a concessão de agradecimentos aos orientadores por parte dos orientandos auxilia na criação de um clima amigável entre professor e aluno. Seguidamente, os estudantes são gratos ao orientador por este ajudá-los a conseguir o título desejado e por meio do amparo nas disciplinas, na dissertação ou na tese e em outras pesquisas. Os pós-graduandos ainda apresentam sentimento de gratidão em relação ao orientador devido ao apoio e ao cuidado oferecido pelos professores e pelo empréstimo de materiais úteis para a execução das pesquisas (Unswortha et al., 2010). Unswortha et al. (2010) identificaram que a gratidão expressa pelos estudantes faz com que orientadores se sintam satisfeitos e contentes consigo mesmo, reconhecidos e realizados com o seu trabalho. Ademais, expressar gratidão melhora a relação de trabalho entre orientadores e orientandos, pois comunica respeito pelo orientador por parte do aluno. A expressão de gratidão acarreta em consequências positivas para estudantes e professores, pois aumenta o afeto positivo e otimiza a relação de trabalho. Contudo, expressar a gratidão não é comum no ambiente acadêmico. Unswortha et al. (2010) sugerem que é preciso pesquisar por que isso acontece.

A relação orientador-orientando muitas vezes não ocorre como os orientandos gostariam. Nesse caso, estes devem gerir a sua relação com o professor: aceitam a situação, não importando o quão ruim está ou tomam uma posição em relação a essa insatisfação (Krauss \& Ismail, 2010). Conforme os resultados da pesquisa de Krauss e Ismail (2010) com 18 pós-graduandos malaios, na maioria dos casos de decepção com os orientadores, os estudantes aceitam o fato que eles não vão ter o que desejam de uma supervisão e que devem ser flexíveis e se ajustarem à situação. Os participantes acreditam que quem dita o ritmo são os supervisores, e os estudantes precisam se adaptar.

No Brasil, o fato de pós-graduados não concluírem o mestrado ou o doutorado, também decorre, principalmente, do estabelecimento de uma relação insatisfatória entre orientador e orientado. Seguidamente, estudantes de pós-graduação brasileiros não estão contentes com a qualidade da orientação que recebem ou com o tempo de orientação destinado a eles pelo orientador (Velho, 2005). Em uma pesquisa realizada no Brasil com 18 enfermeiros mestrandos, a relação com o orientador foi descrita de maneira ambivalente, pois, ao mesmo tempo em que os discentes sentiam-se cobrados por eles, também esperavam que os orientadores estivessem sempre disponíveis (Bujdoso \& Cohn, 2008). Todavia, os padrões de feedback adotados pelos orientadores de pós-graduandos brasileiros não são conhecidos, assim como quais as características que os discentes de pós-graduação do país julgam ser essenciais em seus orientadores. Acredita-se que o conhecimento desses aspectos viabilizará a realização de um trabalho de conscientização com os professores sobre a importância do estabelecimento de uma relação gratificante com seus orientandos para a diminuição das taxas de evasão na pós-graduação. 
Também carece de aprofundamento da literatura as opiniões dos mestrandos e dos doutorandos brasileiros sobre a influência das relações com os pares e com os familiares no processo de adaptação à pós-graduação. Sobre os pares, foi localizado um estudo nacional (Cssarin \& Oliveira, 2012), no qual os colegas são vistos como fontes de troca de informações e materiais bibliográficos, o que, por sua vez, associa-se a um melhor desempenho acadêmico. No que se refere ao apoio dos familiares, não se identificou informações nos estudos nacionais. Porém, acredita-se que a principal tarefa deles, especialmente dos pais, seja a oferta de apoio instrumental, por meio da ajuda financeira, para que o estudante possa concluir o mestrado ou o doutorado.

A partir dos estudos internacionais, percebe-se que as necessidades dos alunos de pós-graduação não são muito diferentes daquelas apresentadas por alunos em outros níveis de formação. Pós-graduandos precisam de apoio contínuo, não apenas de seus supervisores, mas também de seus colegas, amigos e familiares (Tobbell \& O'donnell, 2013). Os estudantes tendem a buscar ajuda de colegas e professores, principalmente do orientador, para informações específicas sobre a vida acadêmica e o programa de pós-graduação, e a família e os amigos para auxílio na resolução de problemas de ordem emocional (Hopwood, 2010; McAlpine, 2012). Essas constatações são semelhantes ao que Teixeira et al. (2008) perceberam em relação à rede de apoio de graduandos do Brasil. Entretanto, não há subsídios para afirmar que isso também ocorre com estudantes de pós-graduação brasileiros.

\section{Fatores institucionais da adaptação à pós-graduação}

Acredita-se que, para ter sucesso na pós-graduação, assim como em outros níveis de formação, é importante que o estudante consiga responder às exigências universitárias e se integrar academicamente (Feldt et al., 2011). Apesar da responsabilidade de formação e adaptação ao curso escolhido ser individual, nota-se que certas características contextuais das universidades facilitam a habituação do aluno ao ambiente acadêmico (Igue et al., 2008). Por exemplo, a ausência de uma orientação em relação aos processos burocráticos universitários é percebida como um dos principais obstáculos à adaptação ao meio universitário, já que dificulta a ambientação à instituição e suas rotinas (Teixeira et al., 2008). É importante que os discentes estejam envolvidos com o curso e comprometidos com a universidade para se adequarem ao ambiente acadêmico (Almeida \& Ferreira, 1999; Baker \& Siryk, 1984).
Quando o estudante ingressa na graduação, as primeiras exigências universitárias burocráticas, como confirmação de vaga, matrícula, carteiras estudantis, podem ser percebidas como severas e fazer com que alguns acadêmicos se sintam perdidos frente ao cotidiano universitário (Teixeira et al., 2008). Gardner (2010) identificou que, na pós-graduação, a maioria dos estudantes também desconhece a estrutura dos programas, bem como os regulamentos e os documentos para concluir o mestrado ou o doutorado. Além disso, os pós-graduandos entrevistados por Gardner (2010) relevaram que gostariam de entender claramente o que o programa de pós-graduação espera que eles executem até o final dos seus cursos, ou seja, quais são as exigências que eles devem atender, e de compreender como ocorre o processo de elaboração da tese antes de se depararam com essa etapa. A autora sugere que conceder essas informações é papel da instituição a fim de evitar que os discentes sintam-se ansiosos devido à falta de conhecimento sobre o que fazer, quais documentos elaborar e como agir. Além disso, os resultados da pesquisa revelaram que os programas de pós-graduação com maiores taxas de evasão foram aqueles que não apresentaram um ambiente de apoio (Gardner, 2010).

Sabe-se que além do ambiente e da cultura organizacional da instituição repercutir no desenvolvimento dos estudantes, os aspectos estruturais, como tamanho e condições dos prédios e laboratórios, e recursos eletrônicos disponíveis também influenciam no desempenho dos universitários (Igue et al., 2008; Teixeira et al., 2008). McAlpine (2012) identificou que as bibliotecas, os laboratórios, os softwares disponíveis na universidade e os recursos tecnológicos ajudam os pós-graduandos a concluírem a pós-graduação, uma vez que eles necessitam do suporte material para a execução de suas pesquisas. Provavelmente, esses discentes não teriam condições financeiras para arcar com os gastos da compra de maquinários, aparelhos e programas indispensáveis para o bom andamento do estudo (McAlpine, 2012).

Por outro lado, os participantes da pesquisa de McAlpine (2012) relataram encontrar dificuldades para expor problemas de desadaptação, uma vez que não sabiam a quem recorrer para realizar esse relato. Ao se deparar com essa realidade, diversas universidades norte-americanas criaram programas de adaptação à universidade. Esses programas contemplam sessões estruturadas para a concessão de informações sobre o ambiente acadêmico e intervenções clínicas, no caso 
de estudantes que apresentam dificuldade no ajustamento a um novo contexto educacional (Mattanah et al., 2010).

O estudo de Hyun et al. (2006) revelou que há um aumento dos pós-graduandos que procuram serviços de aconselhamento devido a dificuldades acadêmicas, problemas emocionais, indecisões em relação à carreira, problemas de relacionamento e preocupações financeiras. Quase três quartos dos participantes tinham conhecimento sobre o serviço de aconselhamento oferecido pela instituição. Entretanto, parece que os estudantes que mais precisam de ajuda não buscam aconselhamento. Por isso, as universidades devem investir na divulgação de programas de aconselhamento para os mestrandos e doutorandos, uma vez que a maioria dos pós-graduandos tende a não frequentar, seguidamente, salas de estudos e a administração de seus cursos. Ademais, é importante divulgar esses serviços de forma a minimizar os preconceitos institucionais e o estigma relacionado à utilização deles, os quais contribuem para a baixa aderência dos pós-graduandos aos programas de aconselhamento.

Também é relevante que as instituições de ensino superior auxiliem seus estudantes a estabelecerem vínculos satisfatórios com os atores envolvidos com o contexto universitário. Isso pode ser realizado por meio do investimento em formas alternativas de socialização. O programa de pós-graduação da Faculdade de Negócios de uma universidade do oeste dos Estados Unidos se dedica a promover a habituação com o ambiente acadêmico por meio da criação de um grupo na rede social Facebook, no qual são postados textos cujos títulos são, por exemplo, "não tenha medo de fazer perguntas" e "artigos demoram a serem publicados" (Ryan, Magro, \& Sharp, 2011). O grupo experimental na rede social, realizado com alunos de doutorado, indicou que a ação aumentou o intercâmbio de conhecimento entre os pós-graduandos por meio do fornecimento de informações sobre o programa e outros aspectos da vida acadêmica, por exemplo, como preparar a dissertação e conferências. Somado a isso, aliviou a apreensão relacionada ao fato de o doutorando ter um novo contexto de convivência a partir do apoio mútuo entre colegas. O estudo concluiu que os grupos do Facebook podem facilitar a troca de conhecimento sobre a estrutura, etapas e cronograma dos programas de pós-graduação e permitir a diminuição da ansiedade a partir do compartilhamento de uma variedade de experiências e do fornecimento de sugestões sobre como enfrentar situações difíceis.
A ação também ajudar a combater o isolamento social dos estudantes por meio de interações e da construção de uma comunidade (Ryan et al., 2011).

Percebe-se que a adaptação a contextos educacionais é definido tanto pela troca entre as expectativas, habilidades e demais características dos estudantes, quanto pelos componentes da universidade: sua comunidade, sua estrutura e elementos organizacionais (Igue et al., 2008). Em relação à realidade de pós-graduação brasileira, acredita-se que é possível que as taxas de evasão estejam mais associadas à cultura institucional dos programas de pós-graduação do que às características pessoais dos discentes. A falta de uma comunidade de referência, tanto intelectual quanto social, que propicia o trabalho isolado do estudante, de conhecimento e de discussão sobre as normas e expectativas do programa e da instituição, de informação prévia sobre o conteúdo dos cursos e de orientação sobre o mercado de trabalho futuro tende a fazer com que pós-graduandos desistam de seus cursos (Velho, 2005).

\section{Considerações Finais}

Esta pesquisa teve como objetivo investigar exploratoriamente os fatores pessoais, relacionais e institucionais que são descritos na literatura como influências no processo de adaptação de estudantes de pós-graduação stricto sensu aos seus cursos. Constatou-se que a integração com a vida de pós-graduando depende, principalmente, do estabelecimento de uma relação gratificante entre orientador e orientando, ambientação com as normas e demandas do programa de pós-graduação e segurança financeira.

Acredita-se que a principal contribuição deste estudo é o fato de ele sistematizar o que já é conhecido sobre o processo de adaptação de mestrandos e doutorandos aos cursos de pós-graduação. É possível que ele minimize uma lacuna teórica existente nos trabalhos sobre adaptação acadêmica, já que esses, normalmente, atentam-se ao processo de adaptação de graduandos e, raramente, pesquisam a integração de pós-graduandos ao ambiente acadêmico. Todavia, os artigos considerados para a realização deste estudo não objetivavam compreender a adaptação de pós-graduandos de maneira integral. As pesquisas atentavam-se para algum dos aspectos pessoais, relacionais ou institucionais inerentes ao processo de ajustamento. Assim, recomenda-se que sejam executadas pesquisas que pretendam entender a adaptação de estudantes de pós-graduação na sua completude. 
Além disso, por este estudo ter revisado pesquisas internacionais, algumas questões da pós-graduação brasileira permanecem sem um entendimento efetivo; por exemplo, a interferência do orientador, dos pares e da família ainda carece de entendimento no cenário brasileiro. Também é desconhecido o motivo pelo qual pós-graduandos do país decidem cursar mestrado ou doutorado, uma vez que as bolsas de estudos são escassas e os salários de muitos docentes de ensino superior não são satisfatórios. Dessa forma, sugere-se que sejam realizadas pesquisas sobre adaptação com mestrandos e doutorandos brasileiros, uma vez que, apesar das semelhanças, a realidade social, cultural e educacional do país pode ser diferente das realidades apresentados pelos estudos revisados.

\section{Referências}

Almeida, L. S. \& Ferreira, J. A. G. (1999). Adaptação e rendimento acadêmico no ensino superior: fundamentação e validação de uma escala de avaliação de vivências acadêmicas. Psicologia: Teoria, Investigação e Prática, 1, 157-170.

Baker, R. W. \& Siryk, B. (1984). Measuring adjustment to college. Journal of Counseling Psychology, 31, 179-189.

Balbachevsky, E. (2005). A pós-graduação no Brasil: novos desafios para uma política bem-sucedida. In C. Brock \& S. Schwartzman (Orgs.), Os desafios da educação no Brasil (pp. 275-304). Rio de Janeiro: Nova Fronteira.

Bennett, S., Mohr, J., BrintzenhofeSzoc, K. \& Saks, L. V. (2008). General and supervision-specific attachment styles: relations to student perceptions of field supervisors. Journal of Social Work Education, 44(2), 75-94.

Bujdoso, Y. L. V. \& Cohn, A. (2008). Universidade como coping para lidar com o trabalho na assistência do mestrando enfermeiro. Revista de Saúde Pública, 42(2), 273-278.

Cantwell, R, H., Scevak, J. J., Bourke, S. \& Holbrook, A. (2012). Identifying individual differences among doctoral candidates: a framework for understanding problematic candidature. International Journal of Educational Research, 53, 68-79.

Conselho Federal de Educação-CFE (1965). Parecer $n^{\circ}$ 977/65. Definição dos cursos de pós-graduação. Brasilia, DF: Autor.
Coordenação de Aperfeiçoamento de Pessoal de Nível Superior-CAPES (2012). GeoCapes. Recuperado em 23 de junho de 2013, de http://geocapes.capes. gov.br/geocapesds/\#

Costa, A. B. \& Zoltowski, A. P. C. (2014). Como escrever um artigo de revisão sistemática. In S. H. Koller, M. C. P. de Paula Couto \& J. V. Hohendorff (Orgs.), Manual de Produção Científica (pp. 55-70), Porto Alegre: Penso.

Credé, M. \& Niehorster, S. (2012). Adjustment to college as measured by the student adaptation to college questionnaire: a quantitative review of its structure and relationships with correlates and consequences. Educational Psychology Review, 24, 133-165.

Cssarin, H. C. S. \& Oliveira, E. S. (2012). O uso da informação no âmbito acadêmico: o comportamento informacional de pós-graduandos da área de educação. Encontros Bibli: revista eletrônica de biblioteconomia e ciência da informação, 17(1), 169-187.

East, M., Bitchener, J. \& Basturkmen, H. (2012). What constitute positive feedback to postgraduate research students? The student's perspective. Journal of University Teaching and Learning Practice, 9(2).

Egan, R., Stockley, D., Brouwer, B., Tripp, D. \& Stechyson, N. (2009). Relationships between area of academic concentration, supervisory style, student needs and best practices. Studies in Higher Education, 34(3), 337-345.

Faro, A. (2013). Estresse e estressores na pós-graduação: estudo com nestrandos e doutorandos no Brasil. Psicologia: Teoria e Pesquisa, 29(1), 51-60.

Feldt, R. C., Graham, M. \& Dew, D. (2011). Measuring adjustment to college: construct validity of the student adaptation to college questionnaire. Measurement and Evaluation in Counseling and Development, 44(2), 92-104.

Gardner, S. K. (2010). Contrasting the socialization experiences of doctoral students in high -and lowcompleting departments: a qualitative analysis of disciplinary contexts at one institution. The Journal of Higher Education, 81(1), 61-81.

Hoffman, J. C. \& Julie, H. (2012). The academic transitional experiences of master's students at the University of the Western Cape. Curationis, 35(1), 1-8.

Psico-USF, Bragança Paulista, v. 20, n. 1, p. 141-152, jan./abr. 2015 
Holley, K. A. \& Caldwell, M. L. (2012). The challenges of designing and implementing a doctoral student mentoring program. Innovative Higher Education, 37, 243-253.

Hopwood, N. (2010). A sociocultural view of doctoral students' relationships and agency. Studies in Continuing Education, 32(2), 103-117.

Hyun, J. K., Quinn, B. C., Madon, T. \& Lustig, S. (2006). Graduate student mental health: needs assessment and utilization of counseling services. Journal of College Student Development, 47(3), 247-266.

Igue, E. A., Bariani, I. C. D. \& Milanesi, P. V. B. (2008). Vivência acadêmica e expectativas de universitários ingressantes e concluintes. Psico-USF, 13(2), 155-164.

Ives, G. \& Rowley, G. (2005). Supervisor selection or allocation and continuity of supervision: ph.D. students' progress and outcomes. Studies in Higher Education, 30(5), 535-555.

Kernan, W., Bogart, J. \& Wheat, M. E. (2011). Health related barriers to learning among graduate students. Health Education, 111(5), 425-445.

Krauss, S. E. \& Ismail, I. A. (2010). Ph.D. students' experiences of thesis supervision in Malaysia: managing relationships in the midst of institutional change. The Qualitative Report, 15(4), 802-822.

Lo Bianco, A. C., Almeida, S. S., Koller, S. H. \& Paiva. V. (2010). A internacionalização dos programas de pós-graduação em Psicologia: Perfil e metas de qualificação. Psicologia: Reflexão e Crítica, 23 (suplemento 1), 1-10.

Maia, D. (2014, 26 de janeiro). Falta de bolsas faz mestrado atingir $70 \%$ de evasão. Folha de São Paulo.

Malagris, L. E. N. et al. (2009). Níveis de estresse e características sociobiográficas de alunos de pós-graduação. Psicologia em Revista, 15(1), 184-203.

Mattanah, J. F., Ayers, J. F., Brand, L. B., Brooks, L. J., Quimby, J. L. \& Scot, M. W. (2010). A social support intervention to ease the college transition: Exploring main effects and moderators. Journal of College Student Development, 51(1), 93-108.

McAlpine, L. (2012). Identity-trajectories: Doctoral journeys from past to present to future. Australian Universities' Review, 54(1), 38-46.
Menandro, P. R. M., Tourinho, E. Z., Bastos, A. V. B. \& Yamamoto, O. H. (2013). Graduate and undergraduate studies: Neighbors without affinity? Paidéia, 23(55), 187-196.

Noy, S. \& Ray, R. (2012). Graduate students' perceptions of their advisors: Is there systematic disadvantage in mentorship? The Journal of Higher Education, 83(6), 876-914.

Polydoro, S. A. J., Primi, R., Serpa, M. N. F., Zaroni, M. M. H. \& Pombal, K. C. P. (2001). Desenvolvimento de uma escala de integração ao ensino superior. PsicoUSF, 6(1), 11-17.

Prepiczka, M. \& Balkin, R. S. (2010). Relationship between wellness and age, matriculation, and relationship status of Counselor Education Doctoral Students. Journal of Humanistic Counseling, Education and Development, 49, 203-215.

Ryan, S. D., Magro, M. J. \& Sharp, J. H. (2011). exploring educational and cultural adaptation through social networking sites. Journal of Information Technology Education: Innovations in Practice, 10, 1-16.

Santos, A. F. \& Alves Júnior, A. (2007). Estresse e estratégias de enfrentamento em eestrandos de ciências da saúde. Psicologia: Reflexão e Crítica, 20(1), 104- 113.

Schwartz-Mette, R. A. (2009). Challenges in addressing graduate student impairment in academic professional psychology programs, Ethics \& Behavior, 19(2), 91-102.

Souza, M. S. (2010). Relação entre suporte familiar, saúde mental e comportamentos de risco em estudantes universitários. Acta colombiana de Psicologia, 13(1), 143-154.

Steiner, J. E. (2005). Qualidade e diversidade institucional na pós-graduação brasileira. Estudos Avançados, 19(54), 341-365.

Teixeira, M. A. P., Dias, A. C. G., Wottrich, S. H. \& Oliveira, A. M. (2008). Adaptação à universidade em jovens calouros. Revista Semestral da Associação BrasiLeira de Psicologia Escolar e Educacional, 12(1), 185-202.

Terrell, S. R., Snyder, M. M. \& Dringus, L. P. (2009). The development, validation, and application of the Doctoral Student Connectedness Scale. Internet and Higher Education, 12, 112-116.

Tobbell, J. \& O’Donnell, V. L. (2013). Transition to postgraduate study: Postgraduate ecological 
systems and identity. Cambridge Journal of Education, 43(1), 123-138.

Tourinho, E. Z. \& Bastos, A. V. B. (2010). Desafios da pós-graduação em Psicologia no Brasil. Psicologia: Reflexão e Crítica, 23 (suplemento 1), 35-46.

Unswortha, K. L., Turnerb, N., Williams, H. M. \& Piccin-Houle, S. (2010). Giving thanks: the relational context of gratitude in postgraduate supervision. Studies in Higher Education, 35(8), 871-888.
Velho, L. (2005, 27 de junho a 10 de julho). Evasão na pós brasileira: Uma crise em formação? Jornal da Universidade Estadual de Campinas, p. 02.

Recebido em: 06/03/2014 Primeira Reformulação em: 13/08/2014 Segunda Reformulação em: 29/12/2014 Aprovado em: 09/02/2015

Sobre as autoras:

Anelise Schaurich dos Santos é mestranda e bolsista CAPES/DS do programa de pós-graduação em Psicologia da Universidade Federal de Santa Maria (UFSM).

Cláudia Maria Perrone é doutora em Psicologia pela Pontifícia Universidade Católica do Rio Grande do Sul (PUCRS) e professora adjunta do Departamento de Psicologia da Universidade Federal de Santa Maria (UFSM).

Ana Cristina Garcia Dias é doutora em Psicologia Escolar e do Desenvolvimento pela Universidade de São Paulo (USP) e professora adjunta do Departamento de Psicologia da Universidade Federal de Santa Maria (UFSM).

Contato com as autoras:

Rua Serafim Valandro, 1637/apto 1004 - Centro - Santa Maria/RS

CEP: $97.015-631$

E-mail: anelise_ssantos@hotmail.com 